\title{
Détermination des stades de maturité sexuelle chez le poisson Galeoides decadactylus (petit capitaine) de la pêcherie artisanale maritime de Grand-Lahou (Côte d'Ivoire).
}

\author{
Kouassi Sylvain Konan *1, Yao Nicolas Amon², Moustapha Diaby 3, Konan N'da4. \\ 1'Département aquaculture, Centre de Recherches Océanologiques BpV 18 Abidjan, Côte d'Ivoire ; \\ Email : kokouasy@yahoo.fr \\ 2Université Peleforo Gon Coulibaly, UFR-Sciences biologiques, Département de Biologie Animale, Bp 1328 Korhogo, \\ Côte d'Ivoire Côte d'Ivoire ; Email : amonyao@yahoo.fr \\ ${ }^{3}$ Agro-pastoral Management Institute, Péléforo Gon Coulibaly University Bp 1328 Korhogo, Côte d'Ivoire ; \\ Email : diae50@yahoo.fr \\ 4UFR Sciences de la Nature, Université Nangui Abrogoua 02 Bp 801Abidjan, Côte d'Ivoire ; \\ Email : konannd65@gmail.com \\ Auteur correspondant ; kokouasy@yahoo.fr ; (+225) 08-76-74-38 ; Bp V 18 Abidjan. \\ Original submitted in on $28^{\text {th }}$ February 2020. Published online at www.m.elewa.org/journals/ on $31^{\text {st }}$ May 2020 \\ https://doi.org/10.35759/JABs.149.6
}

\section{RESUME}

Objectif : La maitrise de la reproduction chez les poissons passe par la connaissance de leurs stades de maturité sexuelle.

Méthodologie et résultats : Les coupes histologiques ont été réalisées au niveau des gonades des poissons capitaines Galeoides decadactylus capturés sur le littoral de Grand-Lahou. Au total,648 spécimens de Galeoides decadactylus composés de 420 mâles et 228 femelles ont permis d'identifier 6 stades de développement des ovaires et 4 stades au niveau des testicules. Les ovaires au stade microscopique I présentent des ovocytes primaires avec un noyau centrale, alors que le stade II regorgent d'ovocytes pré-vitellogéniques et le stade III, des ovocytes en vitellogenèse primaire avec apparition de vésicules vitellines, quand le stade IV présente des ovocytes en vitellogenèse secondaire. Le stade V indique des ovocytes en vitellogenèse avec des vacuoles et inclusions et les ovaires au stade VI montrent des ovocytes en atrésie. Au niveau des testicules, le stade I contient des spermatogonies à forme ovale et le stade II montre des spermatocytes possédant également des noyaux plus petits. Le stade III présente des spermatides avec des noyaux entourés par une fine couche cytoplasmique qui débouchent au stade IV avec des spermatozoïdes encore plus petits que les spermatides.

Conclusion et application des résultats : Ces résultats constituent un socle pour une gestion durable des stocks ainsi que l'expériementation futur de l'élevage du petit capitaine.

Mots clés : Galeoides decadactylus ; ovaires ; testicules. 
Konan et al., J. Appl. Biosci. 2020 Détermination des stades de maturité sexuelle chez le poisson Galeoides decadactylus (petit capitaine) de la pêcherie artisanale maritime de Grand-Lahou (Côte d'Ivoire).

\section{Determination of the sexual maturity stages of threadfin fish Galeoides decadactylus from the artisanal maritime fishery of Grand-Lahou (Côte d'Ivoire).}

\section{ABSTRACT}

Objective: The control of fish reproduction requires knowledge of their sexual maturity stages.

Methodology and results: The histological sections were carried out on the gonads of threadfin fish Galeoides decadactylus captured on the coastal of Grand-Lahou. A total of 648 specimens of Galeoides decadactylus composed of 420 males and 228 females let to identify 6 stages of ovarian development and 4 stages in the testicles. The ovaries at microscopic stage I present primary oocytes with a central nucleus, while stage II is full of pre-vitellogenic oocytes and stage III, oocytes in primary vitellogenesis with the appearance of vitelline vesicles, when stage IV presents oocytes in secondary vitellogenesis. Stage V indicates oocytes in vitellogenesis with vacuoles and inclusions and the ovaries in stage VI show oocytes in atresia. For the testicles, stage I contains oval-shaped spermatogonia and stage II shows spermatocytes also having smaller nuclei. Stage III presents spermatids with nuclei surrounded by a thin cytoplasmic layer, which emerge in stage IV with spermatozoa even smaller than spermatids.

Conclusion and application of results: These results constitute a foundation for the sustainable management of stocks and for the future experience of farming the threadfin.

Keywords : Galeoides decadactylus ; ovaries ; testicles.

\section{INTRODUCTION}

Les ressources de poissons démersaux sont dans une large mesure pleinement exploitées à surexploitées dans la majeure partie du Golfe de Guinée et les poissons de fond constituent environ $10 \%$ du total des exportations de poissons exprimées en valeur (Pulvenis de Séligny et al. 2010). La production maximale soutenable du stock halieutique du littoral ivoirien s'établit à 10 000 tonnes lan avec des captures actuelles stabilisées à 75611 tonnes comportant, entre autres, l'espèce Galeoides decadactylus, uniquement présente sur les côtes ouest africaines
(Motomura. \& Iwatsuki, 2002). Cette espèce est modérément allongé et comprimé, avec une longueur standard d'environ 2,9 à 3,4 fois la hauteur du corps. La bouche est infère avec un museau très court, arrondi et proéminant. On dénombre 8 épines flexibles au niveau de la première nageoire dorsale, une épine flexible et 13 à 14 rayons mous sur la seconde nageoire dorsale. Le corps est argenté et le dos présente une coloration grisâtre, alors que le ventre est blanchâtre.

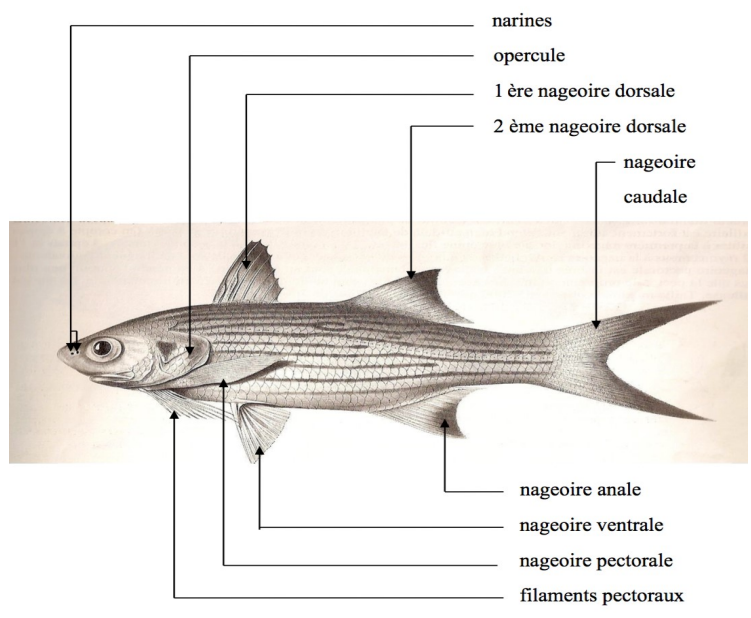


Selon Cochrane ., (2005), la production et le rendement des pêcheries sont limités par un certain nombre de facteurs classés en considérations biologiques, écologiques, socioculturelles, technologiques et économiques. Ainsi, une augmentation de la production nécessite la mise en place d'un plan de gestion durable des ressources. Toutefois, la réalisation des plans d'aménagement s'avère difficile compte tenu $\mathrm{du}$ manque de données biologiques sur les principales espèces. En effet Shoou-Jeng et al., (2007) ont, montré que les informations sur les mécanismes de vie telles que la reproduction des poissons sont très importantes en vue de la préservation de leurs stocks. Or l'étude de la reproduction est basée principalement sur le

\section{MATERIEL ET METHODE}

La reproduction a été étudiée en procédant à des échantillonnages mensuels au sein des captures effectuées par les pêcheurs. Elle a porté sur 648 Galeoides decadactylus composés de 420 mâles et 228 femelles. Les poissons collectés à chaque campagne ont été conservés dans des glacières et ramenés à la salle d'analyse du port d'application du Lycée professionnel de Grand-Lahou. L'identification des sexes et la collecte des gonades mâles et femelles s'est faite après dissection. L'étude des stades microscopiques a consisté à effectuer des coupes histologiques de la gonade. Chaque fragment récolté sur les gonades a été traité au formol $10 \%$ puis

\section{RESULTATS ET DISCUSSION}

La gamétogénèse chez les poissons transforme les cellules primordiales en gamètes. L'évolution de la taille de ces cellules, des noyaux et des inclusions cytoplasmiques a permis de confirmer 6 stades de développement chez les femelles et 4 stades chez les mâles de Galeoides decadactylus.

Description microscopique des gonades femelles (tableau 1)

- Les ovaires au stade microscopique I sont caractérisés par des ovocytes primaires présentant une forme polyédrique et un noyau en position centrale.

- $\quad$ Les ovaires au stade II regorgent des ovocytes pré-vitellogéniques, avec un noyau volumineux contenant de nombreux nucléoles disposés à la périphérie nucléaire. processus de maturation des gonades selon l'évolution de leurs stades de développement macroscopiques ou microscopiques. Le processus de maturation des gonades Galeoides decadactylus présente une particularité due à l'existence d'une inversion sexuelle chez certains individus qui est un hermaphrodisme transitoire (Sidibé, 2003). II précise que l'individu fonctionne comme un mâle pendant qu'il se transforme en femelle, mais qu'à un moment donné l'hermaphrodisme pourrait être fonctionnel. La présente étude est une contribution à la connaissance des stades de maturation microscopiques des gonades de Galeoides decadactylus dont les résultats pourraient servir de base de données pour la gestion de leurs stocks.

conservés par la suite dans de l'alcool $70^{\circ}$. Après la déshydratation dans une série de 7 bains d'éthanol de $70^{\circ}$ à $100^{\circ}$ et la pré-imprégnation dans 3 pots de toluène puis dans de la paraffine liquide de $58^{\circ}$ et $60^{\circ}$, les coupes de 6 à $7 \mu \mathrm{m}$ d'épaisseur ont été effectuées à l'aide d'un microtome mécanique de marque MICRON HM 310. Le déparaffinage des coupes a été effectué dans un bain marie maintenue à $50^{\circ} \mathrm{C}$ puis celles-ci ont été placées dans une étuve à $100^{\circ} \mathrm{C}$. La coloration a été faite à l'hématoxyline-éosine et les tissus ont été collés et recouverts de lamelles, avant de passer à l'examen microscopique à l'aide d'un microscope optique.

- Les ovaires du stade III contiennent des ovocytes en vitellogenèse primaire, avec le noyau et les nucléoles. La corona radiata débute sa mise en place et apparaissent des vacuoles ou vésicules vitellines dispersées dans le cytoplasme.

- Les ovaires au stade IV présentent des ovocytes en vitellogenèse secondaire avec des inclusions à la périphérie du cytoplasme.

- $\quad L e s$ ovaires au stade $V$ indiquent des ovocytes en vitellogenèse avec des vacuoles et inclusions qui occupent tout le volume cellulaire.

- Les ovaires au stade VI montrent des ovocytes hydratés en atrésie. 
Konan et al., J. Appl. Biosci. 2020 Détermination des stades de maturité sexuelle chez le poisson Galeoides decadactylus (petit capitaine) de la pêcherie artisanale maritime de Grand-Lahou (Côte d'Ivoire).

Tableau 1. Aspect microscopique des ovaires de Galeoides decadactylus

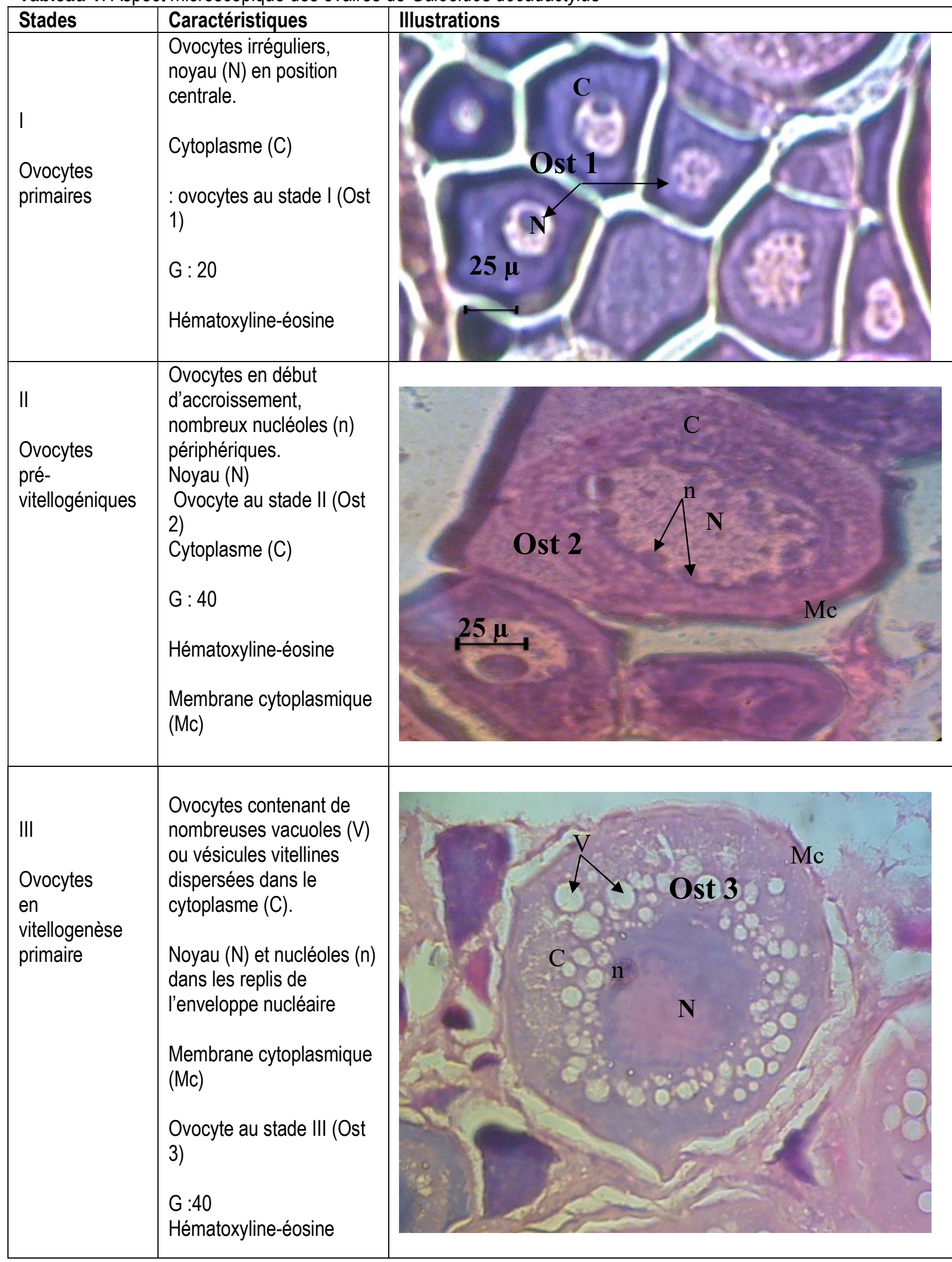


Konan et al., J. Appl. Biosci. 2020 Détermination des stades de maturité sexuelle chez le poisson Galeoides decadactylus (petit capitaine) de la pêcherie artisanale maritime de Grand-Lahou (Côte d'Ivoire).

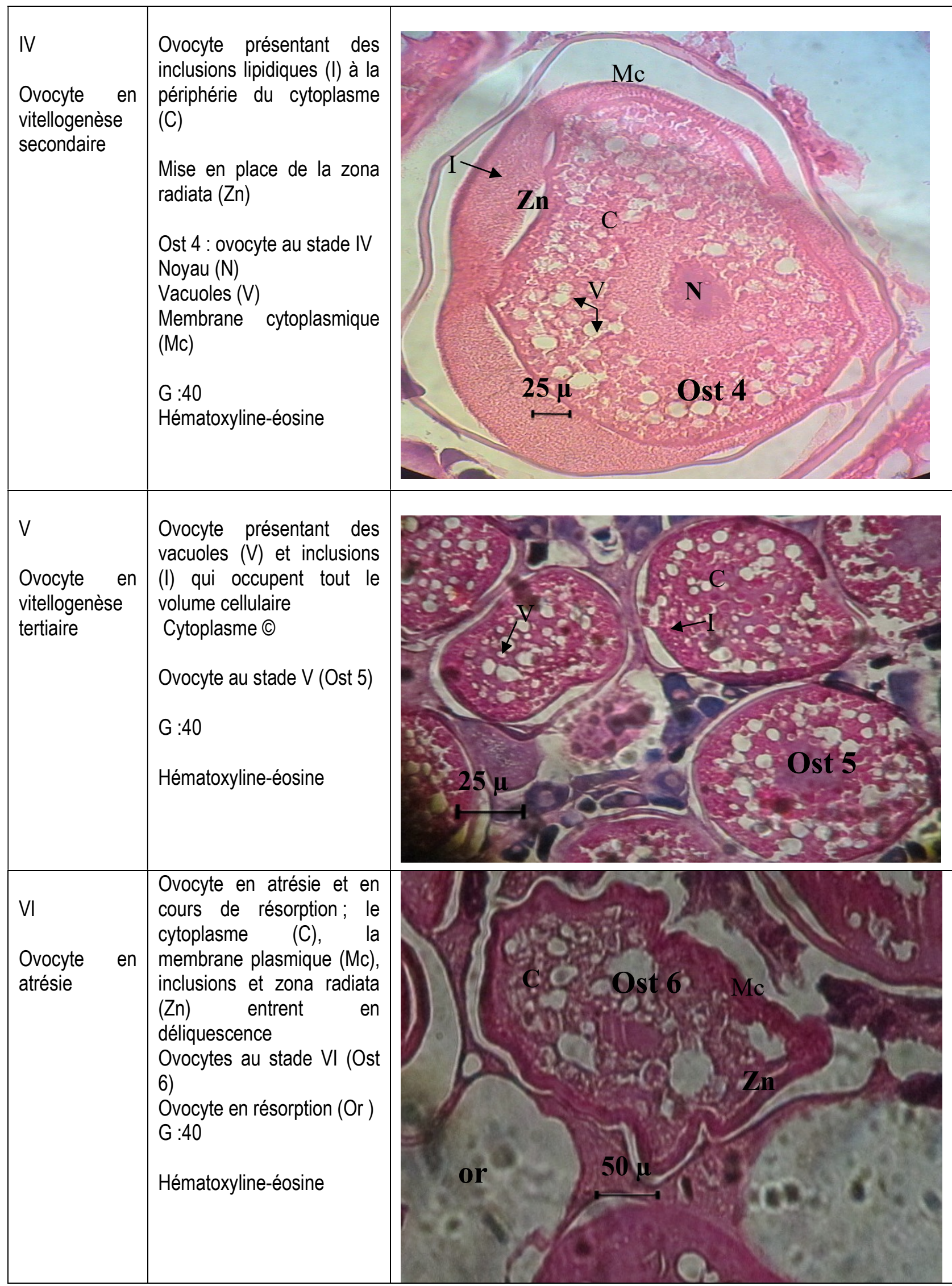


Konan et al., J. Appl. Biosci. 2020 Détermination des stades de maturité sexuelle chez le poisson Galeoides decadactylus (petit capitaine) de la pêcherie artisanale maritime de Grand-Lahou (Côte d'Ivoire).

Description microscopique des gonades mâles (tableau 2)

Les testicules délimitent un réseau conjonctif dans lequel logent les spermatozoïdes. Chaque réseau conjonctif renferme une subdivision de cellules germinales en spermatocytes ou cystes qui parviennent au même stade de développement.

- $\quad$ Les testicules au stade I contiennent dans leur cytoplasme, des spermatogonies à forme ovale possédant de gros noyaux avec un nucléole en position centrale et d'autres à la périphérie.

- Les testicules au stade II présentent des spermatocytes possédant également des noyaux plus petits et plus foncés. Ils sont formés à partir des spermatogonies.

- $\quad$ Les testicules au stade III contiennent des spermatides avec des noyaux entourés par une fine couche cytoplasmique.

- Les testicules au stade IV montrent des spermatozoïdes encore plus petits que les spermatides. Ils sont formés en fin de spermiogénèse et s'identifient facilement grâce à la présence des flagelles et la forte coloration des noyaux.

Tableau 2 : Aspect microscopique des testicules de Galeoides decadactylus

\begin{tabular}{|c|l|c|c|}
\hline $\begin{array}{c}\text { Stades } \\
\begin{array}{c}\text { Testicule au } \\
\text { stade } \\
\text { I }\end{array}\end{array}$ & $\begin{array}{l}\text { Testicule en début de } \\
\text { développement sexuel } \\
\text { spg: spermatogonies } \\
\mathrm{G}: 100 \\
\text { Hématoxyline-éosine }\end{array}$ \\
$\begin{array}{c}\text { Testicule au } \\
\text { stade } \\
\text { II }\end{array}$ & $\begin{array}{l}\text { Testicule en développement } \\
\text { sexuel avancé } \\
\text { spc: spermatocytes } \\
\text { spd: spermatides } \\
\text { tc: tissus conjonctif } \\
\text { Cytoplasme (C) } \\
\text { Cyste (Cyst) } \\
\text { G:40 } \\
\text { Hématoxyline-éosine }\end{array}$ \\
\hline
\end{tabular}



decadactylus (petit capitaine) de la pêcherie artisanale maritime de Grand-Lahou (Côte d'lvoire).

Tabeau 2 (suite)

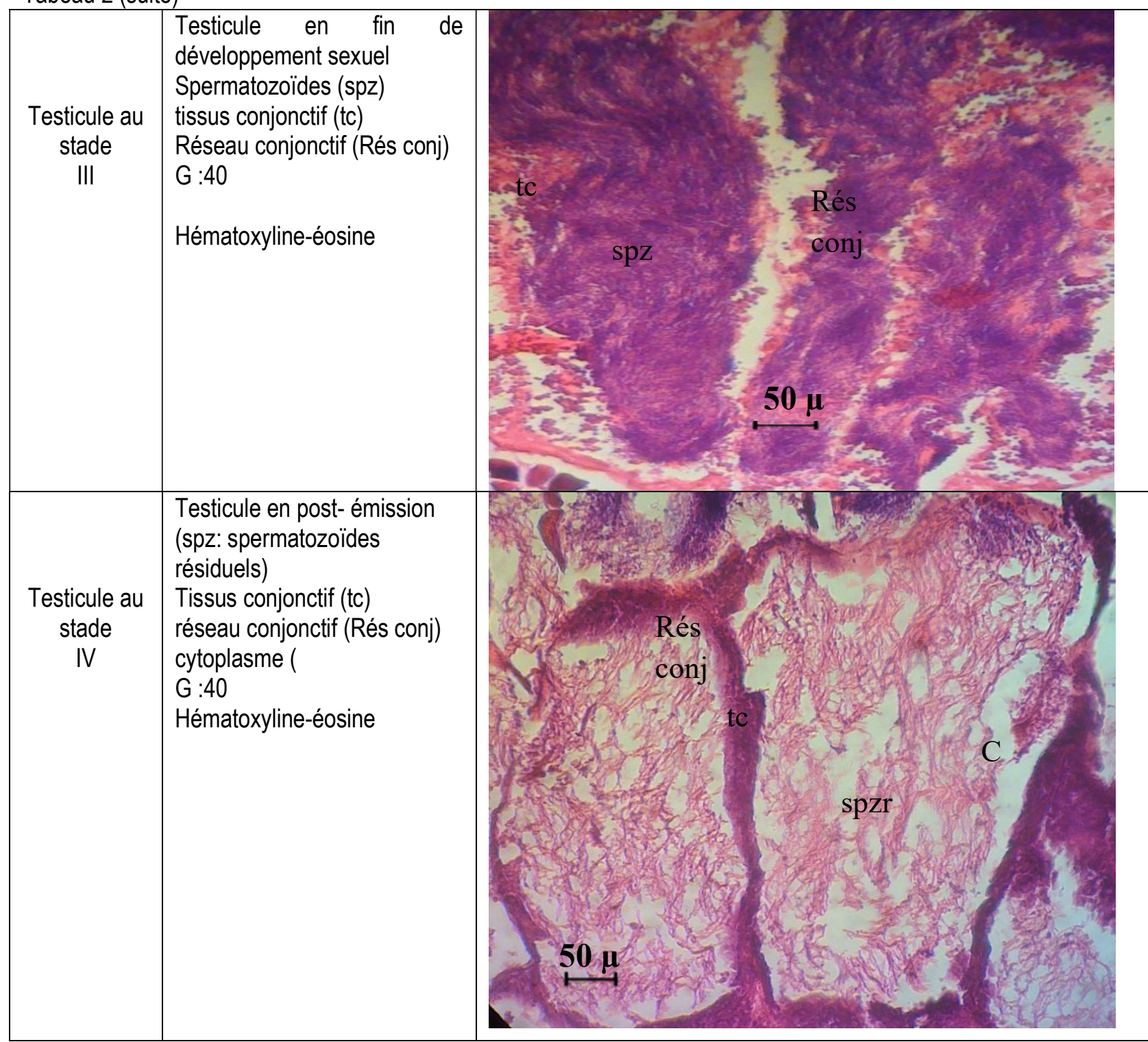

Les ovaires des spécimens de Galeoides decadactylus observés dans la présente étude ont permis d'identifier six stades de développement des gonades chez les femelles de cette espèce. Par contre, quatre stades ont été observés au niveau de leurs mâles. Ces résultats corroborent ceux de Loubens (1966) et de Fontana (1969), relatifs aux capitaines. S'inspirant des observations en zone tropicale, ces auteurs affirment que plusieurs stades sexuels peuvent se superposer, et suggèrent qu'un fractionnement de la ponte peut se produire plusieurs fois au cours d'une même saison de reproduction. Pendant cette dernière, les ovaires contiennent plusieurs classes d'ovocytes évoluant par vagues successives vers la ponte. Ce processus est identique chez les mâles (Loubens, 1966). Ainsi, l'étude des stades de maturités des gonades de ces poissons devra principalement se baser sur une étude microscopique pour mieux appréhender les stades réels de maturité sexuelle. Les auteurs Loubens (1966), Fontana (1969) et de Sidibé (2003) décrivent des stades permettant l'identification des ovocytes qui comportent les stades immatures (I) disposant d'ovocytes transparents, à gros noyau sphérique avec nucléoles en position centrale, ainsi qu'un début maturation (II) avec des ovocytes présentant de gros noyaux avec nucléoles en position excentrique. Les ovocytes entament leur maturation à partir du stade pré-ponte (III) qui indique des ovocytes mûrs 
sphériques, translucides, à membrane épaisse avec des noyaux peu volumineux et la mise en place de la zona radiata. Quant la ponte (IV) montre de gros ovocytes avec de petits noyaux et l'épaississement de la zona radiata. Les ovocytes opaques en atrésie avec des granulations et vacuoles en désagrégation s'observent à la maturation avortée (V). Le stade de repos sexuel $(\mathrm{VI})$ intervient enfin et présente des ovocytes transparents de petite taille avec de hématomes granuleux. Aussi, ils ont décrit les stades et caractères histologiques d'identification des testicules qui ont permis de définir le stade immature (I) indiquant des spermatogonies de petite taille, à gros noyaux et la

\section{CONCLUSION}

La connaissance des stades de maturités des gonades du petit capitaine; Galeoides decadactylus s'est principalement basée sur une étude microscopique pour mieux appréhender les stades réels de maturité sexuelle. Elle a permis de définir 6 stades d'évolution des ovocytes comportant les stades immatures (I), de début maturation (II), de pré-ponte (III), de ponte (IV), de maturation avortée $(\mathrm{V})$ et du stade de repos sexuel (VI). Les stades d'évolution des testicules ont maturation (II) avec des spermatocytes de petite taille et spermatides rondes, puis de petit cytoplasme. Le stade de la spermiation (III) présente des spermatozoïdes avec flagelles et le stade de repos sexuel (IV) indique un canal déférent bien individualisé mais vide et le reste de la gonade est constitué par un tissu lacuneux, anarchique, avec un début de spermatogénèse. En effet, plusieurs stades de maturité peuvent cohabiter dans l'ovaire et seule une analyse fine de la structure de l'ovaire (en particulier de la distribution des tailles des ovocytes qu'il renferme) permet une définition et une classification correctes des stades sexuels (Sidibé, 2003).

permis de définir 4 stades dont le stade immature (I), de maturation(II), de spermiation(III) et de repos sexuel (IV), qui assure la transition vers un début de spermatogénèse. Ces résultats concourent à une meilleure compréhension des processus de reproduction $\mathrm{du}$ petit capitaines et permettront d'assurer une gestion durable des stocks ainsi que l'expériementation de leur élevage.

\section{REFERENCES BIBLIOGRAPHIQUES}

Cochrane K. L., 2005. L'aménagement des pêcheries. Département des pêches de la FAO, $19 \mathrm{p}$.

Fontana A., 1969. Étude de la maturité sexuelle des sardinelles Sardinella eba (Val.) et Sardinella aurita (Val.) de la région de Pointe-Noire. sciaénidés. Thèse de doctorat halieutique ENSA-Rennes, 320p.

Cahiers ORSTOM, séries Océanographie, (2) : 101-114.

Loubens G., 1966. Biologie de polydactylus quadrifilis dans le bas Ogooué (Gabon). Thèse de Doctorat à la faculté des sciences de l'Université de Paris. $163 \mathrm{p}$.

Motomura, K. \& Iwatsuki, (2002): Threadfins of the World, FAO Species Catalogue for Fishery Purposes No. 3, pp 65- 71.

Pulvenis De Seligny J-F., Grainger R. \& Gumy A., 2010. La situation mondiale des pêches et de l'aquaculture 2010. Rome, FAO, $224 \mathrm{p}$.

Shoou-Jeng J., Che-Tsung C., Hsian-Hau L., \& KwangMing L., 2007. Age growth, and reproduction of silky shark, Caracharhinus falciformis, in northeastern Taïwan waters. Fisheries Research 90(2008), 78-85.

Sidibe A., 2003. Les ressources halieutiques démersales côtières de la Guinée: exploitation, biologie et dynamique des principales espèces de la communauté à 